\title{
WHAT GOES UP SOMETIMES STAYS UP: SHOCKS AND INSTITUTIONS AS DETERMINANTS OF UNEMPLOYMENT PERSISTENCE
}

Gianni Amisano, Massimiliano Serati

\section{Contents}

1 Introduction 2

2 A structural VAR model $\quad 4$

2.1 The theoretical model and long-run properties 4

$\begin{array}{ll}\text { 2.1.1 Long-run coefficients } & 6\end{array}$

2.1.2 Impact coefficients 6

2.1.3 How to obtain information on the structural parameters 7

$\begin{array}{ll}2.2 \text { How to construct a theory-instigated prior } & 7\end{array}$

3 Estimation results and comments $\quad 8$

3.1 Demand shocks: the "real power" of the unions as a determinant of hysteresis 9

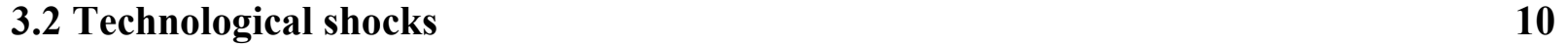

$\begin{array}{ll}\text { 3.3 Participation shocks } & 10\end{array}$

3.4 Permanent changes in the exogenous variables 11

4 Conclusions $\quad 13$

5 Appendix $\quad 14$

$\begin{array}{ll}5.1 \text { Estimation and simulation issues } & 14\end{array}$

$\begin{array}{ll}\text { 5.1.1 The estimated model } & 14\end{array}$

$\begin{array}{ll}\text { 5.1.2 The prior distribution } & 15\end{array}$

$\begin{array}{ll}\text { 5.1.3 Posterior simulation } & 16\end{array}$

$\begin{array}{ll}5.2 \text { Model specification } & 17\end{array}$

$\begin{array}{ll}\text { 5.3 Data definition and sources } & 17\end{array}$ 


\section{Introduction}

Two well-known stylized facts characterised many continental European countries up until the mid-1990s. The first one is the high degree of unemployment persistence. The second one is the sluggishness of the disinflationary process accompanying the rising unemployment. In contrast, the United States and the United Kingdom experienced a more cyclical evolution of unemployment and a lower price stickiness.

These facts have sparked an intense debate among economists on the causes of unemployment, its persistence, and of its cross-country differentials. The issue of persistence is very important since it can be interpreted, together with inflation stickiness, as signalling shifts in the NAIRU. Coherently with this view, many studies have interpreted the European unemployment phenomenon as "structural", pointing out the relevance of labour market rigidities and the intrusive role of "institutions", such as unemployment benefits schemes and workers protection systems. Bentolila and Bertola [3] use the expression "Eurosclerosis" to describe this phenomenon.

A natural evolution of the Eurosclerosis idea emphasises the relevance of longrun unemployment and combines the role of institutional rigidities with the intrusive activity of the unions as the causes of a hysteresis mechanism which could prolong indefinitely the otherwise temporary effects of aggregate demand perturbations. In accordance with the opinion that unions play a relevant role, a traditional framework for the analysis of hysteresis is the Insider/Outsider model (henceforth I/O) as in Blanchard and Summers [7], Layard, Nickell and Jackman [16] (henceforth LNJ), and Lindbeck and Snower [17]. LNJ construct a model in which the unions, some other rigidity factors, such as unemployment benefits and labour taxes (henceforth $U B$ and $L T$ respectively), and shocks generated on the aggregate demand side interact according to an I/O mechanism, in order to produce potentially persistent unemployment.

Without referring to an I/O framework, in the literature other attempts have been made at analysing the role of interactions between shocks and institutions; see for instance, Blanchard ([4] and [5]), Blanchard e Wolfers [8] and Ljungqvist and Sargent [18].

From the econometric point of view, a valid methodology to investigate the relevance of different shocks is the Structural Vector Autoregression approach (henceforth SVAR), very often based on long-run identifying restrictions as in Blanchard and Quah [6]. For instance, Gamber and Joutz (henceforth GJ, [13]), using a VAR model on real wage changes, real output growth and unemployment, identify a demand shock and two orthogonal supply shocks (productivity and labour supply); Balmaseda et al. [2] (henceforth BDL) improves on GJ's analysis, by extending it to different industrialized countries and allowing for identifying long-run restrictions derived from a theoretical model in the I/O framework. From this point of view, the BDL paper conjugates the LNJ approach with Blanchard and Quah's structural VAR methodology.

In this paper, closely following BDL ([2]), we use a Structural VAR approach to investigate the potential determinants of unemployment persistence using data for the period $1975-1995^{1}$, and for four different OECD countries, namely Italy, Sweden, UK and USA. These countries were selected in order to make our analysis more

${ }^{1}$ We repeat the analysis for the period $1975-1998$. See section (3) for the details. 
informative, since the labour markets we consider have different characteristics: a typically "Eurosclerotic" country (Italy), a Scandinavian country (Sweden) with large but strongly coordinated institutions and very active labour policies and two AngloSaxon countries, USA and UK.

We also partially follow Blanchard ([4] and [5]), Blanchard and Wolfers [8] and Ljungqvist and Sargent [18], in explicitly taking into account the interactions between shocks and institutions. To this end, we introduce some innovations with respect to BDL's paper.

First of all, we explicitly introduce some observable rigidity into the estimated model factors, such us $U B$ and $L T$; this allows us to identify more satisfactorily the unobservable shocks and their effects. In fact, BDL describe the effect of these variables by adopting an indirect approach: they identify the unobservable structural shocks, estimate their dynamic effects and, given the hypothesis that $U B$ and $L T$ changes may be considered as wage-push shocks, interpret their consequences as equivalent to those of positive labour supply shocks or negative productivity shocks. However, both economic theory and the existing empirical literature point out that $U B$ and $L T$ changes cannot be treated in this way. Given that there is widespread concern about the relevance of these factors in accounting for unemployment in many European countries (see for instance Siebert [23]), we think that BDL's strategy of leaving them "hidden" as unobservable structural shocks is of dubious value. In our view, this strategy is exposed to the Faust and Leeper [11] critique, which states that long-run identifying restrictions are unreliable when structural identified shocks can be viewed as aggregates of a larger number of underlying shocks that affect the economy in different ways. For all these reasons, in order to correctly identify the unobservable shocks, we enlarge BDL's SVAR model by including explicit measures of $U B$ and $L T$ as exogenous variables ${ }^{2}$ and by considering the dynamic effects of their changes. It is worth pointing out that the Eurosclerosis literature focusses only on the "level" of institutional factors, which Blanchard and Wolfers [8] use in order to condition on them the dynamic effects of shocks. No attempt has been made so far to analyse the dynamic effects of changes of these institutional variables.

The second innovative aspect of our paper concerns the theoretical reference model: since unemployment dynamics reflect separate movements of labour demand and supply, we provide a more realistic and informative representation of labour force behaviour. In particular, unlike BDL, we link participation to the expected and not the actual real wage; moreover, in order to give a more realistic representation of the hysteresis phenomenon, instead of postulating an instantaneous relationship between unemployment and participation, we model an encouragement/discouragement mechanism in which actual participation is negatively linked to past unemployment behaviour.

Thirdly, we adopt a fully Bayesian approach to estimation. This choice enables us to obtain more precise estimates, to obtain the finite sample posterior distributions of the dynamic properties of the model (impulse response functions, forecasting error

\footnotetext{
2 The reason why we focus only on unemployment benefits and labour taxes and not on other rigidity indicstors, such as the union density or its coverage, is that these indicators are accounted for in the I/O framework of the model.
} 
variance decomposition etc..), and to establish a connection between theory and empirical evidence, by using a theory-instigated prior on the coefficients of the estimated SVAR.

As for the connections of our paper with the literature on the interactions between shocks and institutions, since our goal is to obtain a deeper insight into the propagation of shocks and measure their persistence, we perform a time series SVAR analysis on quarterly data, unlike Blanchard and Wolfers [8] who use a panel data approach based on annual data. For this reason, the shocks that we identify do not coincide with those considered by Blanchard ([4] and [5]) and Blanchard e Wolfers [8]. In fact, we follow a more data-instigated approach where shocks are identified on the grounds of their long-run properties and the data are left to tell us about the effects of these shocks and their relative importance to the series being considered.

Given our aim of assessing the relative importance of shocks and their interactions with institutions, we estimate a barebone model, instead of calibrating a rigorously microfounded one, as Ljungqvist and Sargent [18] do.

The structure of the paper is as follows. In section (2) we discuss our modified and extended version of the BDL model and we briefly describe how to construct our prior distribution. In section (3) we provide a detailed report of the empirical evidence. Section (4) contains the conclusions. Estimation technicalities, model specification details and data definitions and sources are relegated to the Appendix ${ }^{3}$.

\section{A structural VAR model}

\subsection{The theoretical model and long-run properties}

The model described in this section is a modified version of the one contained in BDL [2]. Our main modification is that, following the $\mathrm{I} / \mathrm{O}$ tradition, we include unemployment benefits $(U B)$ and labour taxes $(L T)$ which enter the model as a wage push factor both in the wage $\left(z_{t}^{w}\right)$ and in the participation equation $\left(z_{t}^{l}\right)$. The model is:

$$
\begin{aligned}
A D & : y_{t}=\phi\left(d_{t}-p_{t}\right)+a \theta_{t} \\
A S & : y_{t}=n_{t}+\theta_{t} \\
P S & : p_{t}=w_{t}-\theta_{t} \\
W S & : w_{t}=\left\{w_{t}: n_{t}^{e}=\bar{n}_{t}-z_{t}^{w}\right\} \\
\bar{n}_{t} & =\lambda l_{t-1}+(1-\lambda) n_{t-1}, \\
P E & : l_{t}=\alpha\left(w_{t}-p_{t}^{e}\right)-b u_{t-1}+z_{t}^{l}+\tau_{t} \\
z_{t}^{w} & =\kappa U B_{t}+\mu L T_{t}, z_{t}^{l}=\gamma U B_{t}-\alpha L T_{t} \\
\Delta \theta_{t} & =\varepsilon_{t}^{s}, \Delta d_{t}=\varepsilon_{t}^{d}, \Delta \tau_{t}=\varepsilon_{t}^{l}
\end{aligned}
$$

Equation (2.1), (2.2) and (2.3) are exactly as in the BDL paper: on the aggregate demand $(A D)$ side, output depends on real aggregate demand $\left(d_{t}-p_{t}\right)$ and on productivity $\left(\theta_{t}\right)$. Aggregate supply $(A S)$ depends only on labour $\left(n_{t}\right)$ and on productivity.

${ }^{3} \mathrm{~A}$ more detailed version of the appendix can be obtained at the following URL:www.eco.unibs.it/ _amisano/unemp/unemp_app.pdf. 
Price setting is according to a fixed mark-up mechanism (price setting, or PS equation). The wage setting equation $(W S)$ states that the nominal wage $\left(w_{t}\right)$ is set in advance in order to achieve an expected level of employment which is a convex combination of the previous level of employment and the previous level of participation, as in BDL, but here there is a wage push factor $z_{t}^{w}$ that results from the presence of $U B$ and $L T$. In this way, the expected level of employment $\left(n_{t}^{e}\right)$ is lower than $\bar{n}_{t}$. Note that inserting the wage push factor $z_{t}^{w}$ produces the same effects as allowing $\lambda$ to be time variable. In fact, suppose that $u_{t-1}>0$. In this circumstance, in setting $w$ unions aim at a higher employment level $n_{t}^{e}$ via $\lambda$. If we have an increase in $z_{t}^{w}$, since coeteris paribus salary is bargained at a level that corresponds to a lower level of employment than $\bar{n}_{t}$, this has the same effect as a reduction of $\lambda$ (the weight of the outsiders in wage setting). We believe that this is quite a simple and neat way of incorporating observable measures of labour market distortions, which amounts to accounting for a time-varying $\lambda$.

The participation equation (PE) (2.6) links participation $\left(l_{t}\right)$ to expected after-tax real wage, to past unemployment (discouragement effect), to $U B$ and to a stochastic disturbance $\tau_{t}$. Notice that our PE and WS equations allow us to explicitly take into account the effects of interactions among different institutions.

Expression (2.8) states that supply, nominal demand and participation disturbances evolve like random walks. We call the innovations to $d_{t}, \theta_{t}$ and $\tau_{t}$ aggregate demand, aggregate supply and participation shocks, respectively, with a clear reference to standard macroeconomic analysis.

By combining equations (2.1) to (2.4), we can obtain the following wage equation:

$$
w_{t}=d_{t-1}+\frac{(\phi+a-1)}{\phi} \theta_{t-1}-\frac{1}{\phi} \bar{n}_{t}+\frac{1}{\phi} z_{t}^{w}
$$

This shows that the wage is compatible with a level of employment lower than $\bar{n}_{t}$, due to the wage push factor $z_{t}^{w}$.

Our measure of $L T$ is the effective tax rate on labour, following Mendoza et al. [20]. Also Blanchard and Wolfers [8] find that $U B$ and $L T$ are relevant in amplifying the effects of shocks on unemployment ${ }^{4}$. Similar conclusions are also reached by Nickell [21], who shows that $U B$ and $L T$ (to a lesser extent) affect $u$.

The model can be solved obtaining the following 3 equations:

$$
\begin{gathered}
\Delta(w-p)_{t}=\varepsilon_{t}^{s} \\
\Delta y_{t}=\left(\phi \varepsilon_{t}^{d}+(\phi+a) \varepsilon_{t}^{s}-z_{t}^{w}\right)+\lambda \frac{\left[z_{t-1}^{w}+\Delta z_{t-1}^{l}+(1-\phi-a) \varepsilon_{t-1}^{s}+\varepsilon_{t-1}^{l}-\phi \varepsilon_{t-1}^{d}+\alpha \varepsilon_{t-2}^{s}\right]}{\left(1-(1-b-\lambda) L-b L^{2}\right)} \\
\left(1-(1-b-\lambda) L-b L^{2}\right) u_{t}=z_{t}^{w}+\Delta z_{t}^{l}+(1-\phi-a) \varepsilon_{t}^{s}+\varepsilon_{t}^{l}-\phi \varepsilon_{t}^{d}+\alpha \varepsilon_{t-1}^{s}
\end{gathered}
$$

Note that $(w-p)_{t}, y_{t}, n_{t}$ and $l_{t}$ are all I(1), given the statistical properties of $\theta_{t}, d_{t}$ and $\tau_{t}$, and that $u_{t}$ is stationary, i.e. $n_{t}$ and $l_{t}$ are cointegrated. This rules out complete

\footnotetext{
${ }^{4}$ In Blanchard and Wolfers (1999) and Nickell (1997) also the union density variable is found to be relevant. As for the role of $U B$, Blanchard and Wolfers find out that their relevance increases with the length of the benefit duration.
} 
hysteresis and it is therefore important to look at how fast the effects on $u$ of different shocks die out.

\subsubsection{Long-run coefficients}

The system of equations (2.9) to (2.11) can be written in its final form as:

$$
\begin{aligned}
& \mathbf{y}_{t}=\mathbf{C}(L) \mathbf{B e}_{t}+\mathbf{D}(L) \mathbf{x}_{t}, \mathbf{y}_{t}=\left[\begin{array}{c}
(w-p)_{t} \\
y_{t} \\
\sum_{\tau=1}^{t} u_{\tau}
\end{array}\right], \mathbf{x}_{t}=\left[\begin{array}{c}
U B_{t} \\
L T_{t}
\end{array}\right], \boldsymbol{\varepsilon}_{t}=\left[\begin{array}{c}
\varepsilon_{t}^{s} \\
\varepsilon_{t}^{l} \\
\varepsilon_{t}^{d}
\end{array}\right], \\
& \boldsymbol{\varepsilon}_{t}=\mathbf{B e}_{t}, \mathbf{e}_{t} \sim N I D\left([0], \mathbf{I}_{3}\right)=\left[\begin{array}{c}
e_{t}^{s} \\
e_{t}^{l} \\
e_{t}^{d}
\end{array}\right], \mathbf{B}=\left[\begin{array}{ccc}
\sigma_{s} & 0 & 0 \\
0 & \sigma_{l} & 0 \\
0 & 0 & \sigma_{d}
\end{array}\right]
\end{aligned}
$$

Hence, the long-run responses of the endogenous variables with respect to normalised structural shocks are:

$$
\mathbf{C}(1) \mathbf{B}=\left[\begin{array}{ccc}
\sigma_{s} & 0 & 0 \\
(1+\alpha) \sigma_{s} & \sigma_{l} & 0 \\
-(-1-\alpha+\phi+a) \frac{\sigma_{s}}{\lambda} & \frac{1}{\lambda} \sigma_{l} & -\frac{\phi}{\lambda} \sigma_{d}
\end{array}\right]
$$

As in BDL, note that in the long-run real wage is affected only by productivity shocks and that demand shocks do not influence output. These constraints exactly identify the structural shocks. As for the long-run response to a permanent change in an exogenous variables, we have:

$$
\left[\begin{array}{ll}
\lim _{k \rightarrow \infty} \sum_{s=0}^{k} \frac{\partial(w-p)_{t+s}}{\partial U B_{t}} & \lim _{k \rightarrow \infty} \sum_{s=0}^{k} \frac{\partial(w-p)_{t+s}}{\partial L T_{t}} \\
\lim _{k \rightarrow \infty} \sum_{s=0}^{k} \frac{\partial y_{t+s}}{\partial U B_{t}} & \lim _{k \rightarrow \infty} \sum_{s=0}^{k} \frac{\partial y_{t+s}}{\partial L T_{t}} \\
\lim _{k \rightarrow \infty} \sum_{s=0}^{k} \frac{\partial u_{t+s}}{\partial U B_{t}} & \lim _{k \rightarrow \infty} \sum_{s=0}^{k} \frac{\partial u_{t+s}}{\partial L T_{t}}
\end{array}\right]=\left[\begin{array}{cc}
0 & 0 \\
-\kappa+\gamma & -\mu-\alpha \\
\frac{1}{\lambda} \kappa & \frac{1}{\lambda} \mu
\end{array}\right]
$$

Note that the long-run effects of permanent changes in $U B$ and $L T$ on real wage are zero: the real wage is only affected by productivity shocks. The long-run effects of permanept changes in $U B$ on output can be either positive or negative, depending on whether or not the positive effect on participation $(\gamma)$ predominates over the wage push effect $(\kappa)$. Also the long-run effect on output of a permanent increase in $L T$ is uncertain, given that $\alpha$ could be negative, in the case of a large income effect that overcomes the wage push effect $(\mu)$. The effects of permanent changes in $U B$ and $L T$ on unemployment are positive for positive values of $\kappa$ and $\mu$.

\subsubsection{Impact coefficients}

The impact effects of normalised structural shocks and the impact responses to permanent changes in the exogenous variables are respectively: 
$\mathbf{C}_{0} \mathbf{B}=\left[\begin{array}{ccc}\sigma_{s} & 0 & 0 \\ (\phi+\alpha) \sigma_{s} & 0 & \phi \sigma_{d} \\ (1-\phi-a) \sigma_{s} & \sigma_{l} & -\phi \sigma_{d}\end{array}\right],\left[\begin{array}{ll}\frac{\partial \Delta(w-p)_{t}}{\partial U B_{t}} & \frac{\partial \Delta(w-p)_{t}}{\partial L T_{t}} \\ \frac{\partial \Delta y_{t}}{\partial U B_{t}} & \frac{\partial \Delta y_{t}}{\partial L T_{t}} \\ \frac{\partial u_{t}}{\partial U B_{t}} & \frac{\partial u_{t}}{\partial L T_{t}}\end{array}\right]=\left[\begin{array}{cc}0 & 0 \\ -\kappa & -\mu \\ \kappa+\gamma & \mu-\alpha\end{array}\right]$

\subsubsection{How to obtain information on the structural parameters}

Calling:

$$
\mathbf{C}_{0} \mathbf{B}=\mathbf{C}_{0}^{*}, \mathbf{C}(1) \mathbf{B}=\mathbf{C}^{*}, \mathbf{D}(1)=\mathbf{D}
$$

it is easy to see that we can obtain information on the structural parameters from impact and long-run multipliers as follows:

$$
\begin{aligned}
& \lambda=c_{22}^{*} / c_{32}^{*}, \alpha=c_{21}^{*} / c_{11}^{*}-1, \phi=c_{0,21}^{*} / c_{0,11}^{*}-\alpha, a=-c_{0,31}^{*} / c_{0,11}^{*}-\phi+1, \\
& \kappa=-d_{0,21}^{*}, \mu=-d_{0,22}^{*}, \gamma=d_{0,31}^{*}-\kappa
\end{aligned}
$$

These relationships will be used in order to simulate from the posterior distribution of the structural parameters via direct transformation of the simulation of the SVAR model.

\subsection{How to construct a theory-instigated prior}

In many applications it is difficult to incorporate prior beliefs in the form of an informative prior distribution to be used in Bayesian analysis. In our case we have a theoretical model characterised by a set of structural parameters which we reparameterise as a structural VAR model. Our aim is to use our theoretical model in order to construct a prior distribution for the SVAR parameters. The idea is quite simple and works as follows: let us call $\boldsymbol{\xi}$ the vector of the theoretical model parameters on whose likely values we have some beliefs:

$$
\boldsymbol{\xi}=\left[\begin{array}{llllllll}
\lambda & \alpha & \beta & \phi & a^{\prime \prime} & \kappa & \mu & \gamma
\end{array}\right]^{\prime}
$$

and call $\boldsymbol{\theta}$ the vector containing the first order parameters of the SVAR model we estimate. Clearly, $\theta$ is a function of $\xi$ :

$$
\theta=\theta(\xi)
$$

Suppose we have some prior beliefs concerning. $\xi$ and that these can be put into the form of an informative prior distribution $p(\xi)$. Our idea is to transform this prior into a prior on the parameters $\boldsymbol{\theta}$, using a modification of the proposal contained in Ingram and Whiteman [15]. The technical details are provided in Appendix (5.1). In this way we are able to obtain an informative, theory-based prior distribution to be used in our Bayesian analysis. 


\section{Estimation results and comments}

We carry out the Bayesian estimation (see Geweke, [14]) of a structural VAR model. The estimation details are listed in Appendix (5.1) and the data sources are provided in Appendix (5.3). We conduct inference on different features of the model ${ }^{5}$ :

- the dynamic responses of endogenous variables with respect to structural shocks. We evaluate impulse response functions (IRFs, see figures 3.1 to 3.4 ) and forecasting error variance decompositions (FEVDs, see figures 3.5 to 3.8 ) up to 20 steps ahead (5 years);

- the dynamic responses of endogenous variables with respect to permanent changes in the exogenous variables (see figures 3.9 to 3.12 );

- the structural parameters (see table 3.2).

The sample period we work with (1975.1-1995.4) is limited by the availability of data on $U B$ and $L T$. We have also estimated our model for the extended period 1975.1-1998.4, using historical data for the endogenous variables and projected data for the exogenous variables. The evidence shown in this section refers to the 19751995 estimates and appears to be robust to the extension of the sample. However, we point out the few cases in which there are differences between the results of the two sample periods.

The starting point of our analysis is our reference theoretical model, which is capable of generating: (a) temporary (cyclical) unemployment, due to demand shocks; (b) changes in equilibrium unemployment, i.e. in "structural" long run unemployment (NAIRU), due to institutional rigidity factors; (c) hysteresis phenomena which can blur the distinction between (a) and (b); these phenomena are caused by the interaction between shocks and institutions and among the institutions themselves.

To anticipate some of the conclusions:

- Through our estimated model we obtain multi-faceted empirical evidence, in which the main element is hysteresis. Given this, it is whimsical to try to distinguish between shifts on the Phillips curve due to demand shocks, and shifts of the Phillips curve itself caused by institutions changes.

- The responsiveness of real wage with respect to demand shocks is not necessarily a good indicator of labour market flexibility, since it appears to be almost exclusively driven by supply shocks everywhere.

- The empirical relevance of hysteresis might suggest that the unions have a central role. It is important to emphasise that the traditional indicators of unions strength (density and/or coverage) are not always able to give a precise measure of the capacity of the unions to create (and perpetuate) distortions. This can be said also for other traditional measures of labour market institutions, such as the replacement ratio, the average tax rate and so forth. It is only by combining

\footnotetext{
${ }^{5}$ For the adopted specification see appendix 5.2.
} 
together the institutions and by evaluating their interactions with shocks in a dynamic context, as we do, that it is possible to solve some apparent paradoxes, such as the persistence of unemployment even when the traditional measures reflect moves towards less intrusive institutions (as an example, in the UK we have a steady decrease of $U B, L T$ and union density indicator along the whole 1975-1995 sample period).

- Our approach, through an explicit consideration of the main institutional factors, makes it possible to correctly identify the structural shocks, thus rendering the Faust and Leeper [11] critique irrelevant in our context.

\subsection{Demand shocks: the "real power" of the unions as a determinant of hysteresis.}

It appears that demand shocks play a crucial role in affecting the medium-run behaviour of unemployment. Two facts support this view for all countries: a) AD shocks $\left(\varepsilon^{d}\right)$ generate unemployment reactions which disappear only in the long run ; b) FEVD analysis reveals that $\varepsilon^{d}$ shocks explain a large part of unemployment variability at all horizons ${ }^{6}$. The former fact - persistent effects of demand shocks - recalls a hysteresis mechanism and, more generally, supports the relevance of labour market rigidities even in those countries, like USA, usually considered as "flexible". In comparative terms, however, the effects of AD shocks die out more quickly in the USA (7 quarters) than in all other countries considered (13 quarters for Italy and UK, 16 quarters for Sweden).

The empirical evidence of a ubiquitous low wage sensitivity both to cyclical fluctuations $\left(\varepsilon^{d}\right)$ and to labour market disequilibria $\left(\varepsilon^{l}\right)$ strengthens the argument in favour of hysteresis relevance ${ }^{7}$. In fact, we find that the real wage reacts positively to $A D$ shocks only in the very short run and only in $\mathrm{USA}^{8}$ (see figures 3.3 and 3.4) which is a result common to $\mathrm{BDL}$ and GJ.

At the same time, unlike BDL, we find the absence of counter-cyclical behaviour of wages in all the other countries. We believe that this point is particularly interesting, since it is widely debated in the literature. Our results, which are clearly in line with the theoretical model, can be supported by a different analysis: we have computed sample correlations of output gap and variations of real wages for all the countries we consider in this study. The results ${ }^{9}$ are contained in Table 3.1, and they are perfectly consistent with our findings: real wages do not show signs of countercyclicity.

As a possible explanation of the detected persistence, the hysteresis approach underlines the interaction between long-lasting demand shocks and the power of unions, usually measured by the degree of density (UND), or coverage (UNC). Rather than looking at UND and UNC, we interpret our empirical evidence on the basis of the

\footnotetext{
${ }^{6}$ Only the UK unemployment is significantly affected also by $\varepsilon^{l}$ at least in the short run.

${ }^{7}$ Romer (1996), pag 467, says: "...insider-outsider considerations reduce the cyclical sensitivity of the marginal cost of labour to firms.."

${ }^{8}$ But there are no real wage reactions if we estimate the SVAR over the period 1975-1998.

${ }^{9}$ For each country, we compute the output gap as the difference between actual and HP filtered GDP.
} 
"real power" of the unions, defined as their ability to influence market equilibrium which is hidden in the parameter $\lambda$, but also in its interactions with $L T$. The existence of a positive correlation ${ }^{10}$ between levels of $L T$ and the degree of unemployment persistence (high $L T$ countries are also "highly persistent" countries) supports the idea that $L T$ acts as a factor strengthening the consequences of $\mathrm{AD}$ shocks.

\subsection{Technological shocks}

Our evidence shows that it is where unions are stronger that the positive effects of technological shocks on unemployment are weaker. The medium-long run effects of AS shocks on $u$ are not significant in any country. This fact, which is perfectly in accordance with our theoretical model, is in contrast with part of the previous literature. In particular, Blanchard ([4] and [5]) finds that AS shocks, in the form of a reduction in the growth rate of TFP, are relevant for explaining unemployment in the medium-long run. A possible reason for our different findings could be that our sample starts from 1975, whereas for the industrialised countries the turning point of the total factor productivity growth is in 1973. Moreover, in our insider/outsider model in which wage bargaining is done in order to maximise only insiders' utility, the potentially favourable effects on $u$ of a technological shock are partially dissipated by the increase in real wage.

BDL and GJ find at least short-run positive effects of $\varepsilon^{s}$ on $u$ for all countries they consider, but a similar result occurs in our analysis only for USA (and only for the 1975-1995 estimates) ${ }^{11}$.

More mixed is the evidence on output effects of AS shocks: output increases both in the short and in the long-run, as predicted by the theoretical model, only in Sweden, whereas in USA the effects are significant only in the medium-long run ${ }^{12}$; surprisingly, we find no significant effects at all in Italy and UK. For these countries the dynamic effects of supply shocks on output is very imprecisely estimated.

\subsection{Participation shocks}

When we analyse the effects of participation shocks we have to keep in mind that the theoretical model suggests that positive participation shocks reduce the "real power" of the unions and viceversa. Migration phenomena, population growth and increase in the participation rates do not seem to be useful factors in explaining longrun unemployment movements in the countries being considered. In general, unlike $\mathrm{BDL}^{13}$, participation shocks have no effects on unemployment at any horizon, the only exception being the UK (see figure 3.3 ), where we find a positive effect in the short run.

\footnotetext{
${ }^{10}$ Especially in the second half of our sample.

${ }^{11}$ Gali (1999) finds a short run negative effect of technology shocks on hours.

${ }^{12}$ But they appear significant over the whole simulation horizon, if we look at the estimates obtained on the basis of the "extended" sample (1975-1998). There, the recent productivity-based growth of the USA economy is at work.

${ }^{13}$ BDL (2000) find positive short and medium run effects for most of the countries they consider.
} 
In our view these findings indicate that a positive $\varepsilon_{t}^{l}$ loosens the capacity constraint and triggers an increase in $n$ which matches the increase in participation. Evidence on output is generally consistent with this view ${ }^{14}$ : participation shocks increase output in the long-run in all countries; for Italy, Sweden and USA we have also short-run effects (see figures 3.1 and 3.2$)^{15}$.

In the light of these findings, participation shocks may be viewed as having positive effects on the labour market (in the sense of stimulating more employment). In fact, as is widely known, models with hysteresis (see Blanchard and Summers [7] or Lindbeck and Snower [17]) postulate that insiders can obtain a level of wage higher than the competitive one and still exclude the outsiders only if the following two conditions are met: 1) the outsiders are not competitive (given that human capital depreciates); 2) hiring and firing costs prevent firms from substituting insiders with outsiders. A positive shock on participation adds new workers to the overall labour supply. These workers are obviously not marginalised and this circumstance will moderate insiders' wage requests. This phenomenon should gradually erode the power of the insiders.

Our results point out that participation shocks have no long-run effects on real wages. This is hardly a surprise, since this feature is imposed in order to identify the model. As for short-run effects, the theoretical model indicates that the real wage should not be affected at any horizon by labour supply shocks and our results are consistent with these prescriptions of the theoretical model. Nevertheless, they significantly differ from the ones obtained by BDL, who find that the real wage significantly decreases in the short run for most of the countries they consider. This occurs in particular in France and Germany, but (less strongly) also in USA and UK. In contrast, we find perfect and rapid employment reactions (with the exception of the UK) so that the labour market equilibrium is guaranteed and there are no down-pressures on real wages.

\subsection{Permanent changes in the exogenous variables}

An important issue is whether the included institution variables are relevant per se, or in virtue of their interaction with the "real power" of unions. It would be very useful to be able to say something precise about the role of the institutions. Unfortunately, because of the unsatisfactory quality of the data on these series, our results concerning the role of these variables are often not very clear-cut or easy to interpret. We have a slightly clearer picture on the effects of permanent changes in $L T$.

We must re-iterate that the insertion in the model of observable measures of the institution variables is mainly motivated by the need to correctly identify the structural shocks, without incurring in the Faust and Leeper critique. In fact, our findings suggest that the signs of the long-run responses to $U B$ and $L T$ variations need not coincide, either in the theoretical model or in the estimated systems. Moreover, these variations have effects which are different from those of the structural shocks $\varepsilon^{s}$

\footnotetext{
${ }^{14}$ The FEVDs provide a partial support to this picture: $\varepsilon^{l}$ explains most of the output variance both in the short and in the long run in Italy and USA and at least in the long run in Sweden (in the short run $\varepsilon^{d}$ has a strong relevance). Only UK output behaviour is mainly influenced by $\varepsilon^{d}$ at all horizons.

${ }^{15} \mathrm{It}$ is worth recalling that BDL find significant effects on output at all horizons.
} 
and $\varepsilon^{l}$. This casts some doubts on the BDL results, where variations in $U B$ and $L T$ are treated as positive labour supply or negative productivity shocks.

Turning to the details of our findings (see figures 3.9 to 3.12 ), in some cases the estimated responses to changes in $U B$ and $L T$ corroborates our main point, i.e. that the most important factor is the "real power" of the unions. In fact, we have already seen (see section 3.1) that $L T$ is a factor capable of amplifying the effects on $u$ of demand shocks and of strengthening the hysteresis mechanism. Hence, a high level of $L T$ and high degree of real power of the unions jointly produce perverse effects on unemployment ${ }^{16}$.

However, labour taxes appear to be relevant in explaining long-run unemployment in an even more direct way. In fact, in three out of the four countries considered, $L T$ shocks have medium-and long-run effects on unemployment ${ }^{17}$ : in USA we have an increase of unemployment at all horizons (see figure 3.12), whereas in Italy we have an increase in $u$ in the medium run (6 to 12 quarters, see figure 3.9 ). We believe that this evidence could be the signal of positive values for $\mu$ (relevant impact of $\Delta L T$ on wages): in fact, just for Italy and USA, the posterior distribution of $\mu$ (figures 3.13 to 3.16) assigns the most part of the probability to positive values ${ }^{18}$. On the other hand, real wage decreases in USA, both in the short and long-run (see figure 3.12), and remains unchanged in Italy ${ }^{19}$. The USA evidence in this regard is not consistent with the theoretical model, according to which there should be no long-run effects of $L T$ on $(w-p)$ (see expression (2.14)) and could be due to the inability of unions to prevent producers from shifting the tax burden onto workers and consumers (i.e. we have a reduction of $w$ and/or an increase in $p$ ). This is another example of how relevant is the interaction between $L T$ and the role of unions.

In Sweden, given an $L T$ increase, we find a decrease of $u$ in both the short and long-run ${ }^{20}$, while $(w-p)$ increases but only up to the medium run (2 to 15 quarters, see figure 3.10). Both results are hard to explain unless one is prepared to consider $L T$ as not exogenous (given political economy considerations ${ }^{21}$ ).

Turning to the effects of permanent changes of $U B$, also in this case this variable is important bacause of its connection with the unions' role and importance. As for $(w-p)$, there are no effects at any horizon for Italy and Sweden, as suggested by the theoretical model. We have positive long-run effects in USA (see figure 3.12) and negative ones in UK; the explanation for the USA evidence could be the power of non-unionised workers (high job turn-over) which translates an increase in the reservation wage into an effective increase in the real wage. This does not happen in Italy and Sweden where the outsiders' power is probably lower. The UK result is clearly spurious, since the sample correlation between $U B$ and $(w-p)$ is -0.965 .

We find no significant effect on $u$ in any country and at any horizon, bar a very

\footnotetext{
${ }^{16}$ Also Blanchard and Wolfers (2000) emphasise the relevance of similar interaction mechanisms.

${ }^{17}$ In $\mathrm{UK}$, there are no significant effects on $u$ and $w p$.

${ }^{18}$ The opposite occurs for UK and Sweden.

${ }^{19}$ There is only an irrelevant and temporary reduction in the very short run.

${ }^{20}$ The posterior probability of a negative $\mu$ is around 0.95 .

${ }^{21}$ The sample correlation coefficient between $u$ and $L T$ in Sweden for the period 1975-1995 is -0.876 , and the sample correlation between $w p$ and $L T$ is -0.31 .
} 
small negative effect in Sweden ${ }^{22}$. It is difficult to find a sensible explanation for the irrelevance of $U B$ on unemployment. Perhaps this could be ascribed to a statistical problem, like the low time variability of $U B$ in each country, or the interactions among different wage push factors (the role of the unions, $U B$ and $L T$ ) implied by our wage setting equation (2.4). However, the evidence supports the idea of very low values for $\kappa$; in fact, looking at table 3.2, the upper bound of the $80 \%$ posterior confidence interval for $\kappa$ is 0.0015 for USA, 0.0044 for Italy, 0.0047 for UK and 0.0331 for Sweden.

\section{Conclusions}

In this paper we analyse the main determinants of unemployment persistence in four OECD countries in the period 1975-1995. To this end, we have critically revisited a model already used in the literature, which is based on the hypotheses of imperfect competition both on labour and goods markets (I/O approach). The innovative elements of our analysis are the following: (a) we explicitly insert labour market rigidity variables, such as unemployment benefits and labour taxes; (b) we insert a more sensible discouragement mechanism in the labour supply behaviour; (c) we use a Bayesian estimation approach in order to obtain more efficient estimates and to be able to build an informative prior suggested by the theoretical model for the SVAR parameters.

Our empirical evidence on unemployment persistence confirms some common views and is partly consistent with the findings of previous existing literature, but it also introduces new useful elements into the picture. First of all, the common view (see for example Daveri and Tabellini [9]) that the Anglo-Saxon, continental and North-European labour markets differ in terms of their degrees of rigidity is only partly confirmed. In fact, we found the effects of demand shocks to be highly persistent in Sweden, Italy and UK, and less long-lasting in USA. However, in partial accordance with Blanchard and Quah [6], BDL [2] and GJ [13], the FEVD analysis shows that aggregate demand shocks play a dominant role in explaining unemployment fluctuations not only in the short run, but also in the medium/long-run, and also in flexible labour markets like the USA.

As for the country specific features, they seem to be largely determined by different degrees of insider power, confirming unions as a relevant factor influencing unemployment persistence. A less intrusive role of American unions is confirmed by a kind of auxiliary evidence: only in USA do firms appear to be able to shift the burden of higher taxes onto workers and consumers and only in USA does the effective wage increase as a consequence of a rise in the reservation wage. This is the consequence of the great power of non-unionised workers and high job turn-over (low average length of unemployment spells).

As for the effects of $U B$ and $L T$, we find that these variables do not generate identical responses on the observable variables and that they do not have the same effect as positive labour supply or negative productivity shocks. This casts some doubts

\footnotetext{
${ }^{22}$ There is evidence of reverse causation ( $U B$ and $L T$ not fully exogenous) in Sweden, where the interaction among workers, producers, unions and government is different with respect to the other countries and is characterised by a high degree of coordination.
} 
on the validity of BDL approach, where UB and LT are not explicitly introduced in the model. In particular, $L T$ also seems to be able to influence medium/long-run unemployment at least in USA and Italy (and in Sweden, although the sign of the effect is counter-intuitive) through its interaction with the power of the unions; labour taxes are very high in Italy, a high unemployment country and they can be reasonably blamed for the persistence of high unemployment there.,

We also found some interesting similarities among countries; in particular, real wages positively react to productivity shifts everywhere and at all horizons. Moreover, in contrast with previous findings, the real wage responses to aggregate demand shocks is nowhere countercyclical. In addition, real wage is not sensitive to labour supply shocks, so its evolution is not a reliable signal of the labour market conditions.

Another difference with respect to the previous literature (e.g. BDL [2] and GJ [13]) is that we do not find any evidence of short-run positive effects on unemployment as a consequence of a productivity shift; therefore, the role of technological bias as a relevant explanation for short-run unemployment may be excluded.

As for policy implications and developments, we do not intend to propose any miraculous remedy for the European unemployment persistence problem, beyond those contained in the extensive literature on this topic, as it would be a futile operation. Nevertheless, on the basis of our empirical findings, a simple and general, albeit often ignored, methodological rule may be suggested to deal with the problem at hand. The crucial points are the interactions between shocks and institutions, already stressed in Blanchard [4] and Blanchard and Wolfers [8], and, overall, the interactions among the different institutions. The effects on unemployment of changing a single institution depend on the size and the features of all the others. As a consequence, no partial policy measure which acts on a single aspect of the question (for example, benefits), may be effective in solving the problem. Only a general approach, producing a thorough and full reform of labour markets, goods markets and welfare state is bound to get satisfactory results.

Anyway, this paper is only the first attempt to empirically analyse the hysteresis problem with a general equilibrium approach and within a time series framework. Various future lines of research are possible. On the methodological side, important improvements might derive from the estimation of a potentially over-identified structural model based on the use of Markov Chain Monte Carlo (MCMC) techniques. This would allow us to test the structure against the data. Moreover, a statistically sound comparison among different countries could be obtained only by adopting a Panel-VAR framework.

\section{Appendix}

\subsection{Estimation and simulation issues}

\subsubsection{The estimated model}

The model we estimate is a VAR with deterministic and exogenous variables (VARX), which can be considered as the reduced form of our structural model. We are in a situation of exact identification (see Amisano and Giannini [1]). 
In order to describe the estimation approach we follow, via suitable definition of the vector $\mathbf{y}$ and the matrix $\mathbf{Z}$, we write the reduced form VARX model as:

$$
\mathbf{y}=\mathbf{Z} \boldsymbol{\theta}+\boldsymbol{\varepsilon}, \boldsymbol{\varepsilon}=\operatorname{vec} \mathbf{E}, \boldsymbol{\varepsilon} \sim N\left(0, \mathbf{H}^{-1} \otimes \mathbf{I}_{T}\right)
$$

where $\mathbf{E}$ is a $(T \times n)$ error term matrix.

\subsubsection{The prior distribution}

A convenient choice for the prior is a Normal-Wishart distribution:

$$
\boldsymbol{\theta} \sim N\left(\underline{\theta}, \underline{\mathbf{H}}_{-\boldsymbol{\theta}}^{-1}\right), \mathbf{H} \sim W i(\underline{\nu}, \underline{\mathbf{S}})
$$

This prior distribution has the advantage of delivering quite tractable posterior distributions for the parameter of the model and of being simple enough to accommodate reasonable prior beliefs. We start from the VARMA(X) representation of the theoretical model (2.9)-(2.11):

$$
\mathbf{y}_{t}=\mathbf{A}_{1} \mathbf{y}_{t-1}+\mathbf{A}_{2} \mathbf{y}_{t-2}+\mathbf{B}_{0} \mathbf{x}_{t}+\mathbf{B}_{1} \mathbf{x}_{t-1}+\mathbf{B}_{2} \mathbf{x}_{t-2}+\boldsymbol{\Phi}_{0} \varepsilon_{t}+\Phi_{1} \varepsilon_{t-1}+\boldsymbol{\Phi}_{2} \varepsilon_{t-2}
$$

It is easy to show that plausible values for the parameters $\lambda$ and $b$ ensure that the VMA part is fundamental and can be inverted to yield:

$$
\begin{aligned}
\mathbf{A}^{*}(L) \mathbf{y}_{t} & =\mathbf{B}^{*}(L) \mathbf{x}_{t}+\boldsymbol{\varepsilon}_{t} \\
\mathbf{A}^{*}(L) & =\left[\boldsymbol{\Phi}^{*}(L)\right]^{-1} \mathbf{A}(L)=\mathbf{I}_{3}-\mathbf{A}_{1}^{*} L-\mathbf{A}_{2}^{*} L^{2}, \\
\mathbf{B}^{*}(L) & =\left[\boldsymbol{\Phi}^{*}(L)\right]^{-1} \mathbf{B}(L)=\mathbf{B}_{0}^{*}+\mathbf{B}_{1}^{*} L
\end{aligned}
$$

Notice that all the $\mathbf{A}^{*}(L)$ and $\mathbf{B}^{*}(L)$ have finite order and this descends from the unimodularity of the VMA component (see Lutkepohl [19], ch. 7). It is also possible to show that in our case the transformation from the theoretical model parameters to the SVAR model parameters is linear. For this reason, computation of the prior distribution for the SVAR model could be carried out also analytically. The simulation approach that we are about to describe works in this simple framework and it can be applied also to more complicated settings. In order to incorporate theoretical considerations in the prior structure, we partially follow Ingram and Whiteman ([15]): let us collect the first order parameters of the theoretical model presented in section (2) in the vector $\boldsymbol{\xi}$. We have some ideas about the likely values of these parameters and we can represent these ideas stochastically, via a tractable prior distribution:

$$
\boldsymbol{\xi} \sim T N\left(\underline{\boldsymbol{\xi}}, \underline{\mathbf{H}}_{\xi}^{-1}\right)
$$

where $T N$ stands for appropriately truncated normal distribution. The choice of a truncated normal distribution is justified only by its convenience, but it is possible to use other distributions. We can obtain arbitrarily as many IID draws from the prior distributions (5.5) as we want, and map them onto draws from a prior distribution for the first-order parameters $\boldsymbol{\theta}$ of the VAR representation (5.3) of the model:

$$
\boldsymbol{\theta}^{(i)}=\boldsymbol{\theta}\left(\boldsymbol{\xi}^{(i)}\right), \boldsymbol{\xi}^{(i)} \sim T \cdot N\left(\underline{\boldsymbol{\xi}}, \underline{\mathbf{H}}_{\xi}^{-1}\right), i=1,2, \ldots, M
$$


Given these draws, one can compute $\underline{\theta}_{M}$ and $\underline{\mathbf{H}}_{\theta_{M}}$, the first and second moments from these draws. We can then use these moments as moments for a normal prior distribution of $\boldsymbol{\theta}$, which we name TISB (theory instigated, simulation-based prior).

The definition of prior first and second moments for $\boldsymbol{\xi}$ reflects both general theoretical considerations and country-specific features:

- $\phi \in[0, \infty)$; we set its prior mean (for all countries) equal to 0.8 with a standard deviation $(S D)$ of 0.3 .

- $\lambda \in[0,1]$;we set its prior mean to 0.7 for USA and Sweden (high outsider power), to 0.5 for UK and to 0.3 for Italy. In all cases we set $S D=0.10$.

- $\alpha$ :we think it sensible that its value be smaller than 1.0. We set its prior mean to 0.30 and its $S D=0.10$ for all countries.

- $b$ : we set its prior mean to 0.10 for all countries but Italy, where we set it to 0.15 ; the prior $S D$ is set to 0.05 everywhere.

- $\kappa, \mu, \gamma:$ we have the prior belief that these coefficients are small. For the sake of simplicity, we set their prior mean for all countries at the value 0.30 and their $S D$ to 0.10 .

- $a$ is set to 0.30 (in all countries), with standard deviation equal to 0.10 .

The prior settings are summarized in table 5.1 .

\subsubsection{Posterior simulation}

Given the prior, the joint posterior distribution is:

$$
\begin{aligned}
\ln p(\boldsymbol{\theta}, \mathbf{H} \mid \mathbf{Y}, \mathbf{X})= & c+(T / 2+v-1-n) \ln |\mathbf{H}|-\frac{1}{2}\left[(\mathbf{y}-\mathbf{Z} \boldsymbol{\theta})^{\prime}\left(\mathbf{H} \otimes \mathbf{I}_{T}\right)(\mathbf{y}-\mathbf{Z} \boldsymbol{\theta})+\right. \\
& \left.+(\boldsymbol{\theta}-\underline{\boldsymbol{\theta}})^{\prime} \underline{\mathbf{H}}_{\boldsymbol{\theta}}(\boldsymbol{\theta}-\underline{\boldsymbol{\theta}})+\operatorname{tr}(\underline{\mathbf{S H}})\right]
\end{aligned}
$$

which can be easily sampled by means of a simple two-block (one for $\boldsymbol{\theta}$ and one for $\mathbf{H}$ ) Gibbs sampling algorithm ([14]), given the usual conditionally conjugated analysis. It is possible to sample sequentially from these two conditional posterior distributions and to have an arbitrarily large sample ( $M$ draws) of Markov Chain draws from the joint posterior distribution of all parameters of the VARX model:

$$
\boldsymbol{\alpha}^{(i)}=\left[\begin{array}{l}
\boldsymbol{\theta}^{(i)} \\
\operatorname{vech}\left(\mathbf{H}^{(i)}\right)
\end{array}\right], i=1,2, \ldots M
$$

Finally, it is easy to obtain algebraically $M$ draws for whatever function $\gamma^{(i)}$ of $\alpha^{(i)}$ just by applying the required mapping from $\boldsymbol{\alpha}^{(i)}$ to $\gamma^{(i)}$. The posterior distribution of $\gamma$ (and of any continuous function of them) can therefore be easily simulated ${ }^{23}$.

\footnotetext{
${ }^{23}$ We have coded all the necessary computations in Fortran. The routines are available on request.
} 


\subsection{Model specification}

For all countries considered the model we use is a VAR specification with 3 lags in the endogenous variables and the current value and first 2 lags on the exogenous variables. We have introduced a constant term and a very small set of impulse dummies to eliminate some outliers. The complete list of dummy variables is as follows:

Italy: 76Q1, 76Q2, 92Q4; Sweden: 80Q1, 93Q2; UK: 79Q2; USA: no dummies.

\subsection{Data definition and sources}

The source of data is Datastream, except for $U B$ and $L T$, for which the source is the OECD dataset provided to us by S. Scarpetta. All series have been seasonally adjusted and output and wages are expressed in real terms. In the estimation we used their logarithmic transformation (save for $U B$ and $L T$, which are percentage measures).

Series definitions:

- Output: Gross Domestic Product.

- Real wage: ratio between hourly wage rate for the whole economy and GDP deflator. Unemployment: standardised unemployment rate (whole economy).

- Unemployment Benefits: averages of replacement ratios for two earning levels, three family situations and three durations of unemployment ([24]). Quartely data are obtained by interpolation of the annual series.

- Labour taxes: obtained as the ratio between the sum of income taxes, indirect taxes and Social Security Contributions (SSC both on workers and firms) and the sum of average gross wage and SSC on firms ([24]). Quartely data are obtained by interpolation of the annual series. 


\section{References}

[1] Amisano, G. and C. Giannini (1997), Topics in SVAR Econometrics, Springer Verlag, Berlin.

[2] Balmaseda, M., J. Dolado and D. Lopez Salido (2000), "The dynamic effects of shocks to labour markets: evidence from OECD countries", Oxford Economic Papers, 52, 3-23.

[3] Bentolila, S and G. Bertola (1990), "Firing costs and labour demand: how bad is Eurosclerosis?", Review of Economic Studies, 57, 381-402.

[4] Blanchard, O. (1998a), "Revisiting European unemployment. Unemployment, capital accumulation and factor prices", paper written for the Conference in honor of Michael Bruno, Jerusalem, November 1997.

[5] Blanchard, O. (1998b), "Thinking about unemployment", paper written for the Baffi lecture, Rome, October 1998.

[6] Blanchard, O. and Quah, D. (1989), "The dynamic effects of aggregate demand and aggregate supply disturbances", American Economic Review, 79, 655-673

[7] Blanchard, O. and L. Summers (1986), "Hysteresis and the European unemployment problem", in Stanley Fisher ed., NBER Macroeconomics Annual

[8] Blanchard, O. and J.Wolfers (1999), "The role of shocks and institutions in the rise of European unemployment: the aggregate evidence", NBER Working Papers, n. W7282

[9] Daveri.F., Tabellini G. (2000), Unemployment, growth an taxation in industrial countries", Economic Policy: a European Forum, April 2000, 0(30), 47-88.

[10] Doan, T., R. Litterman, C. Sims (1984): Forecasting and conditional projections using realistic prior distributions, Econometric Reviews, 3, 1-100.

[11] Faust, J and Leeper, E.M. (1997), "When do long-run identifying restrictions give reliable results?, Journal of Business \& Economic Statistics, 15, 3, 345-353.

[12] Gali, J. (1999): "Technology, employment and the business cycle: do technology shocks explain aggregate fluctuations?", American Economic Review, 89, 249-71.

[13] Gamber, E. and Joutz, F. (1993), "The dynamic effects of aggregate demand and supply disturbances: Comment", American Economic Review, Papers and Proceedings, 83, 1387-93.

[14] Geweke, J., (1999):"Using simulation methods for Bayesian econometric modelling: inference, development and communication", Econometric Reviews, 18, 1-74. 
[15] Ingram, B.F. and C.H. Witheman (1994): "Supplanting the 'Minnesota' prior : forecasting macroeconomic time series using real business cycle model priors", Journal of Monetary Economics, 34, 497-510.

[16] Layard R, Nickell S, Jackman R, (1991) "Unemployment - Macroeconomic performance and the labour market", Oxford, Oxford University Press.

[17] Lindbeck, A. and D. Snower (1988), "The Insider/Outsider theory of employment and unemployment", MIT Press, Cambridge Mass.

[18] Ljungqvist, L. and T.J. Sargent (1998), "The European unemployment dilemma", Journal of Political Economy, 106, 514-551.

[19] Lutkepohl, H. (1993), "Introduction to Multiple Time Series Analysis", Springer - Verlag Berlin.

[20] Mendoza, E.G., A. Razin, L.L. Tesar (1994): "Effective tax rates in macroeconomics", Journal of Monetary Economics, 34, 297-323.

[21] Nickell S. (1997), "Unemployment and labor market rigidities: Europe versus North America", Journal of Economic Perspectives, 11 (3), 55-74.

[22] Romer, D. (1996), Advanced macroeconomics, McGraw-Hill, New York.

[23] Siebert, H. (1997): "Labor market rigidities: at the roots of unemployment in Europe", Journal of Economic Perspectives, 11(3), 37-54.

[24] Scarpetta, S. (1996), "Assessing the role of labour market policies and institutional settings on unemployment: a cross-country study", OECD Economic Studies, 26, 43-98. 

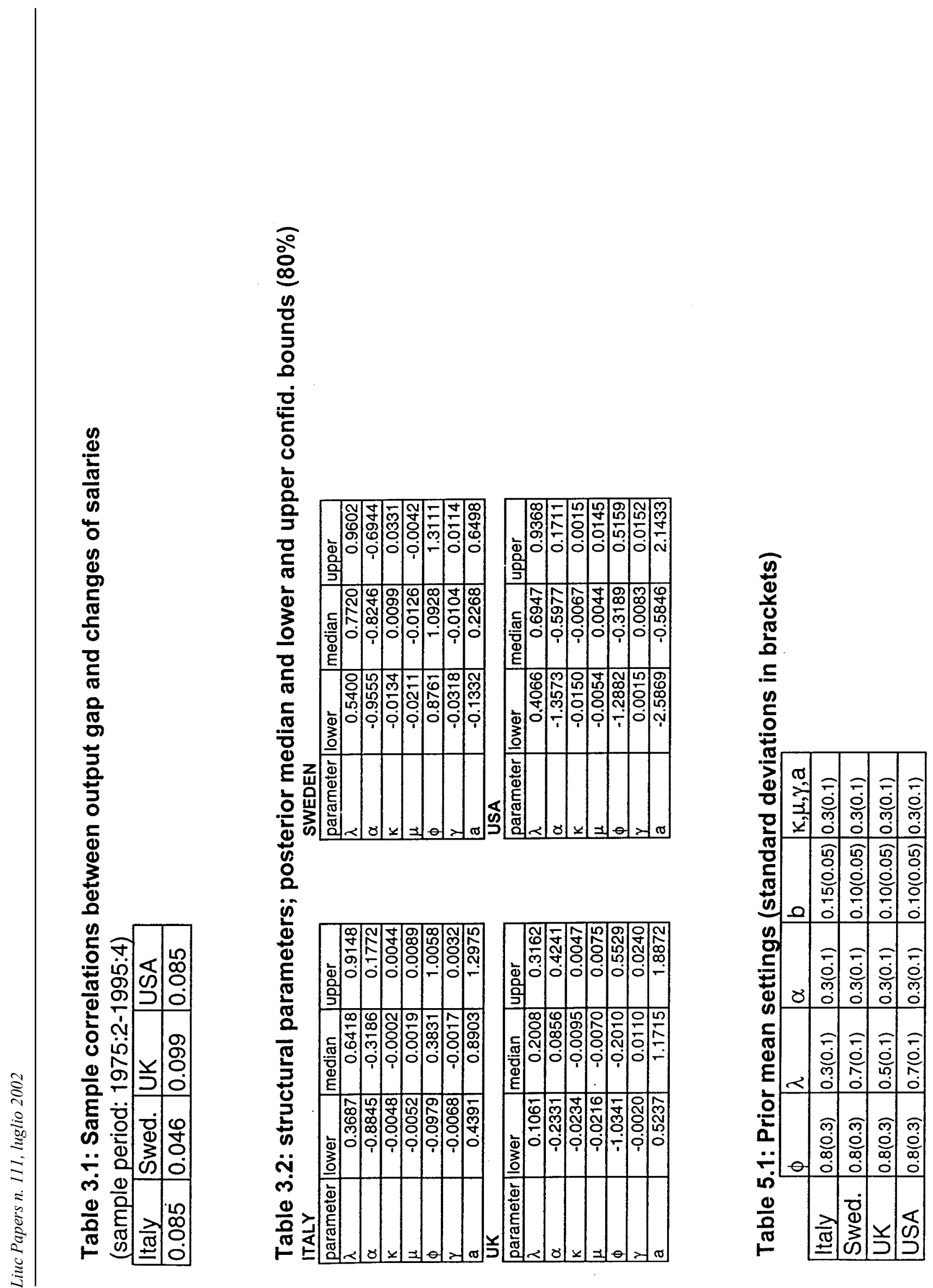

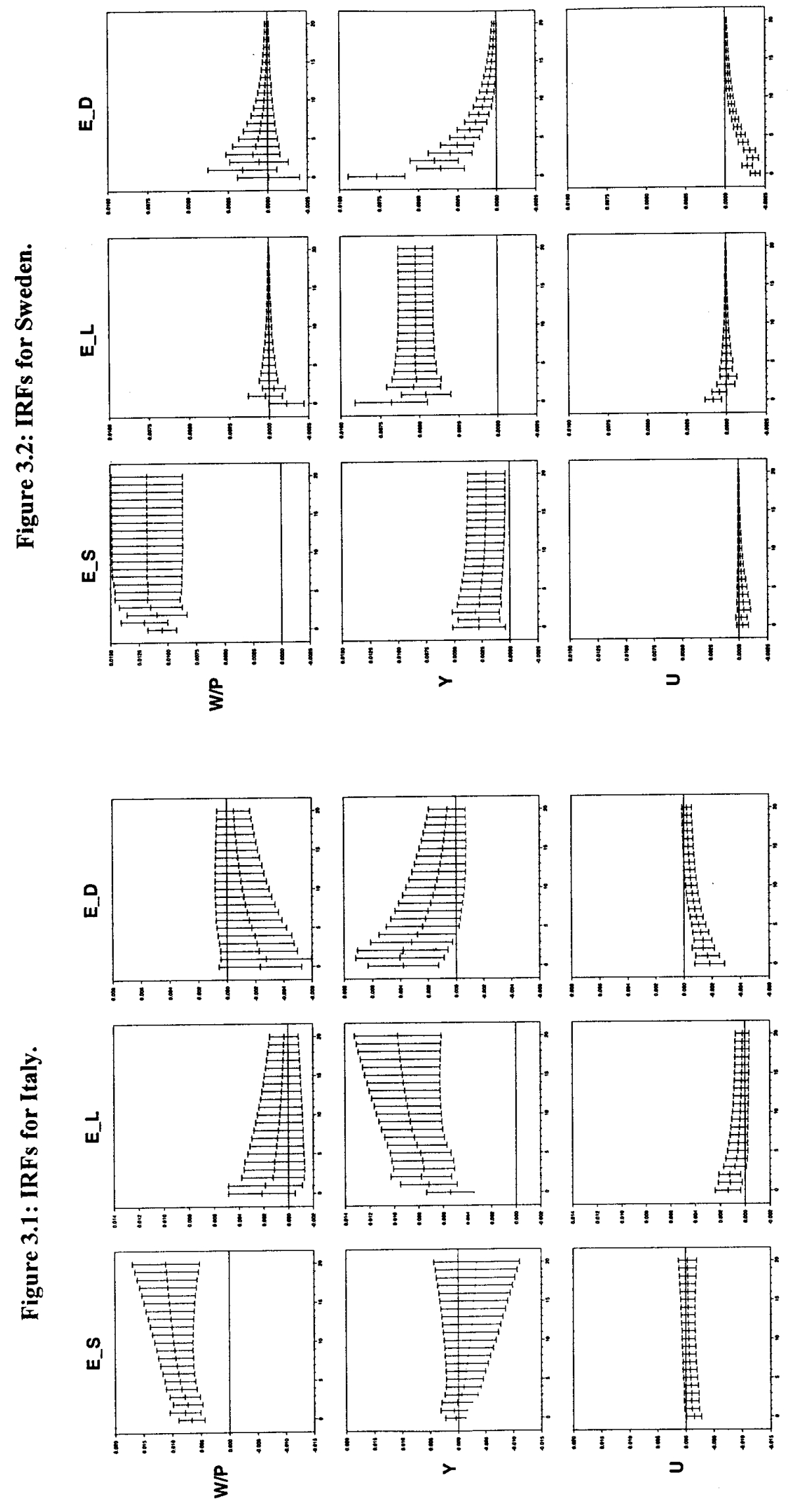

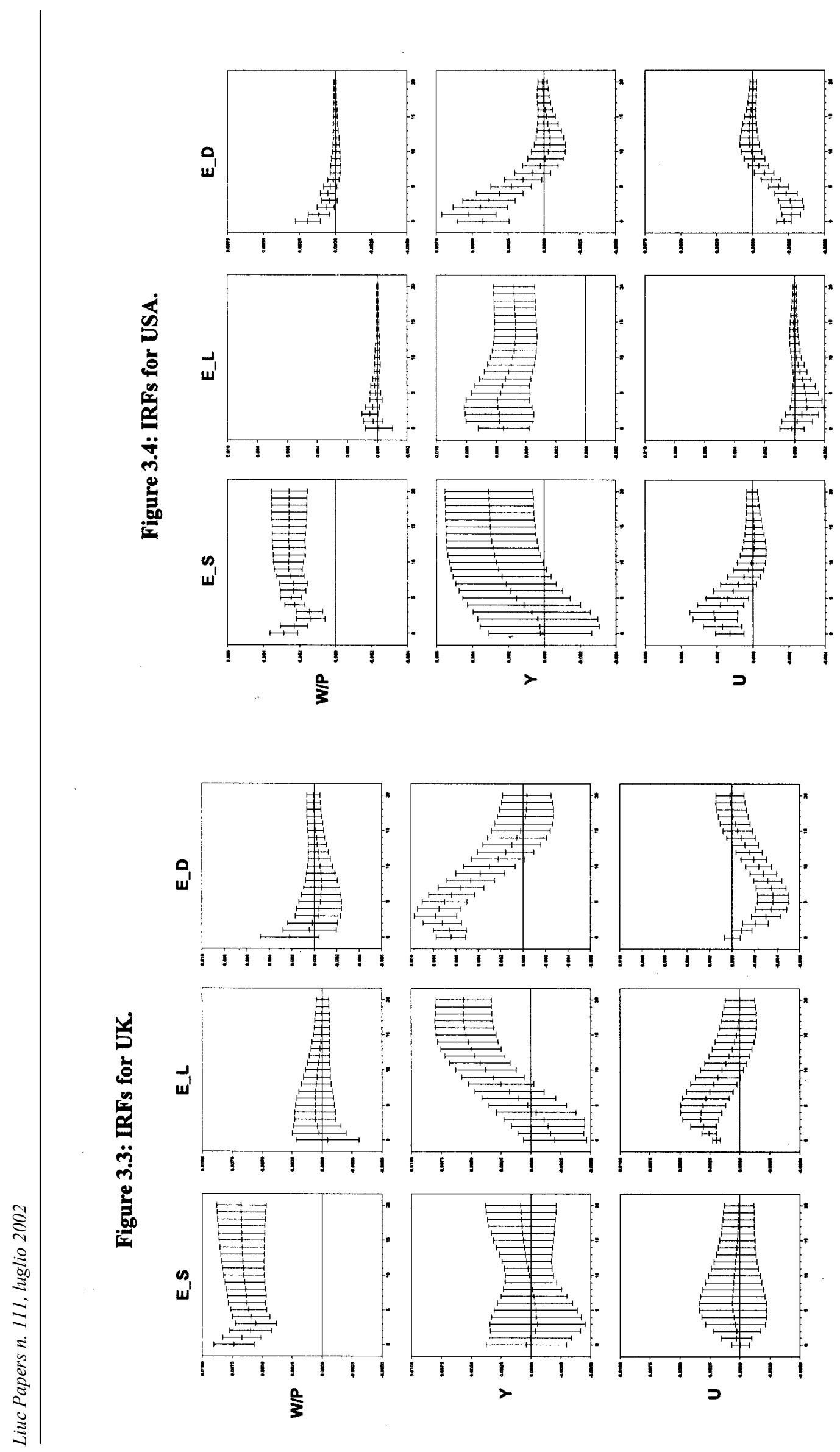

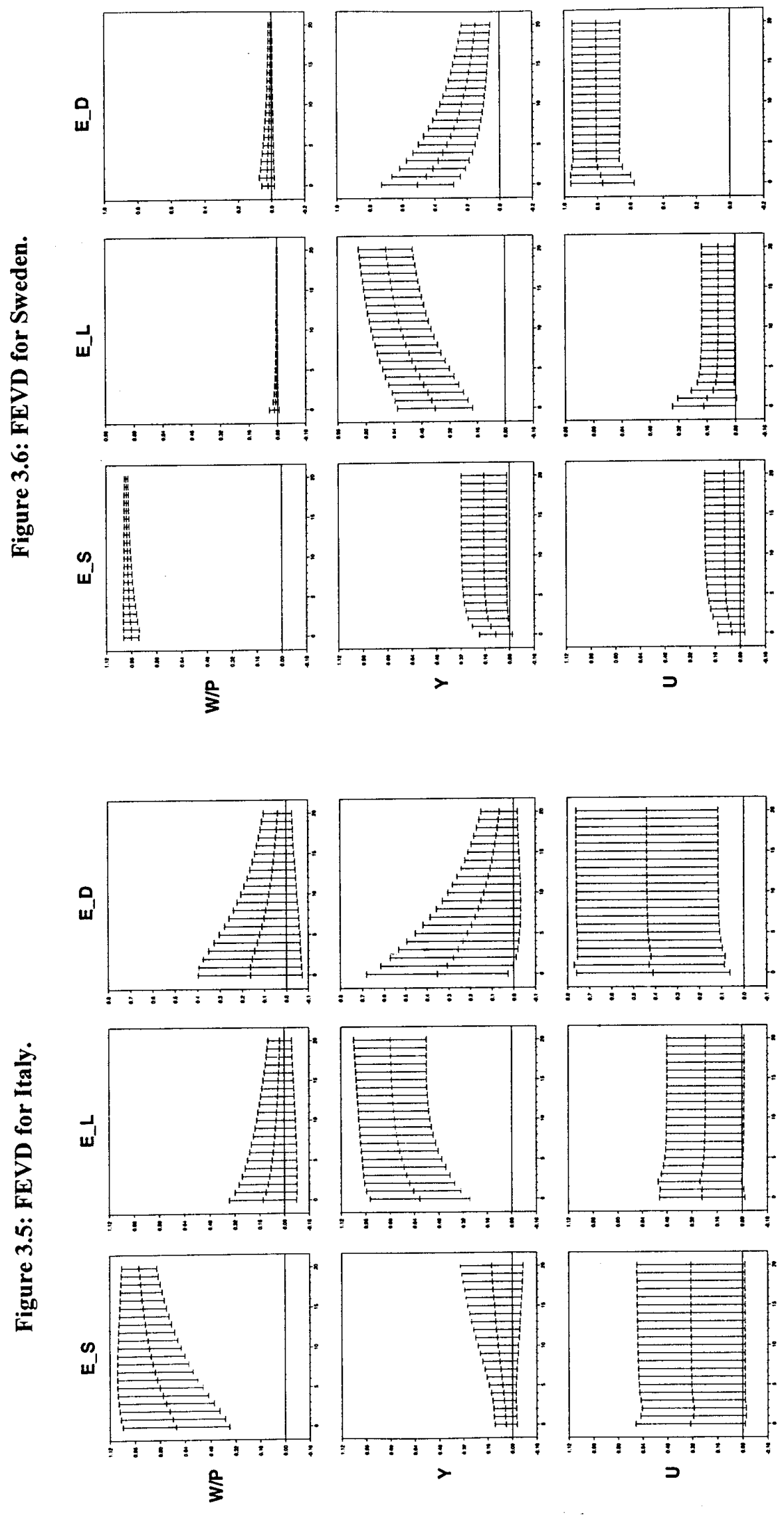


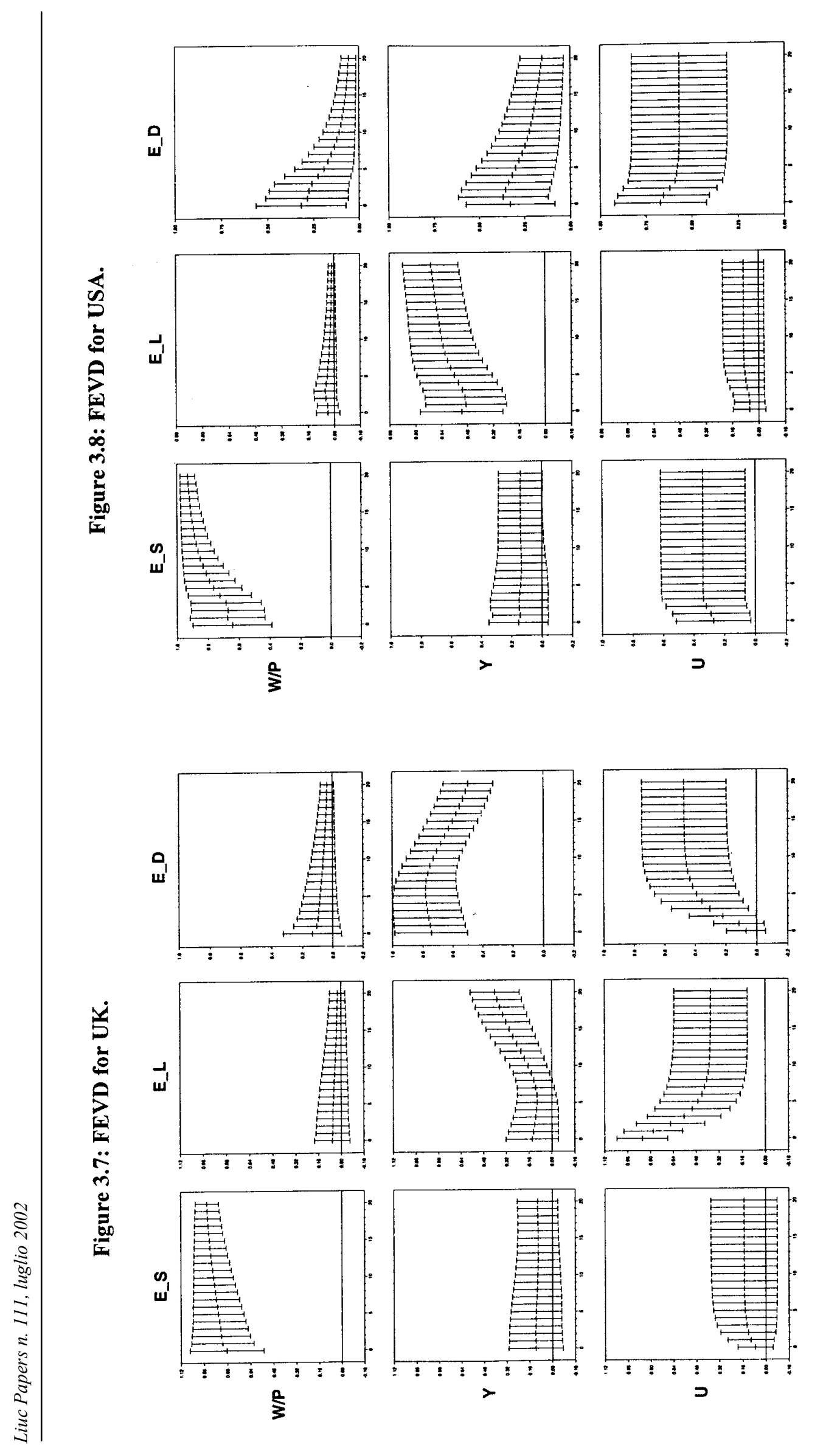



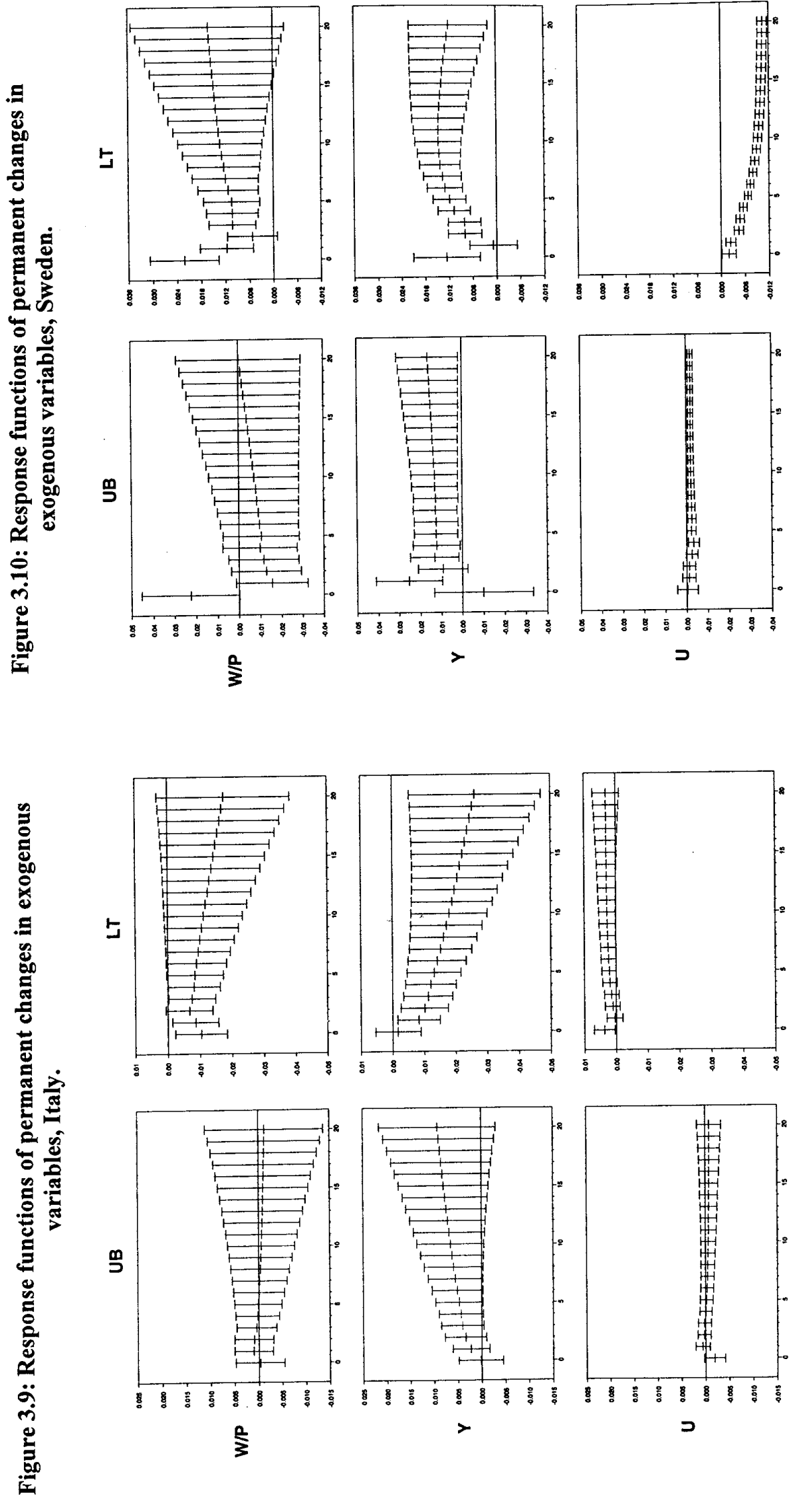


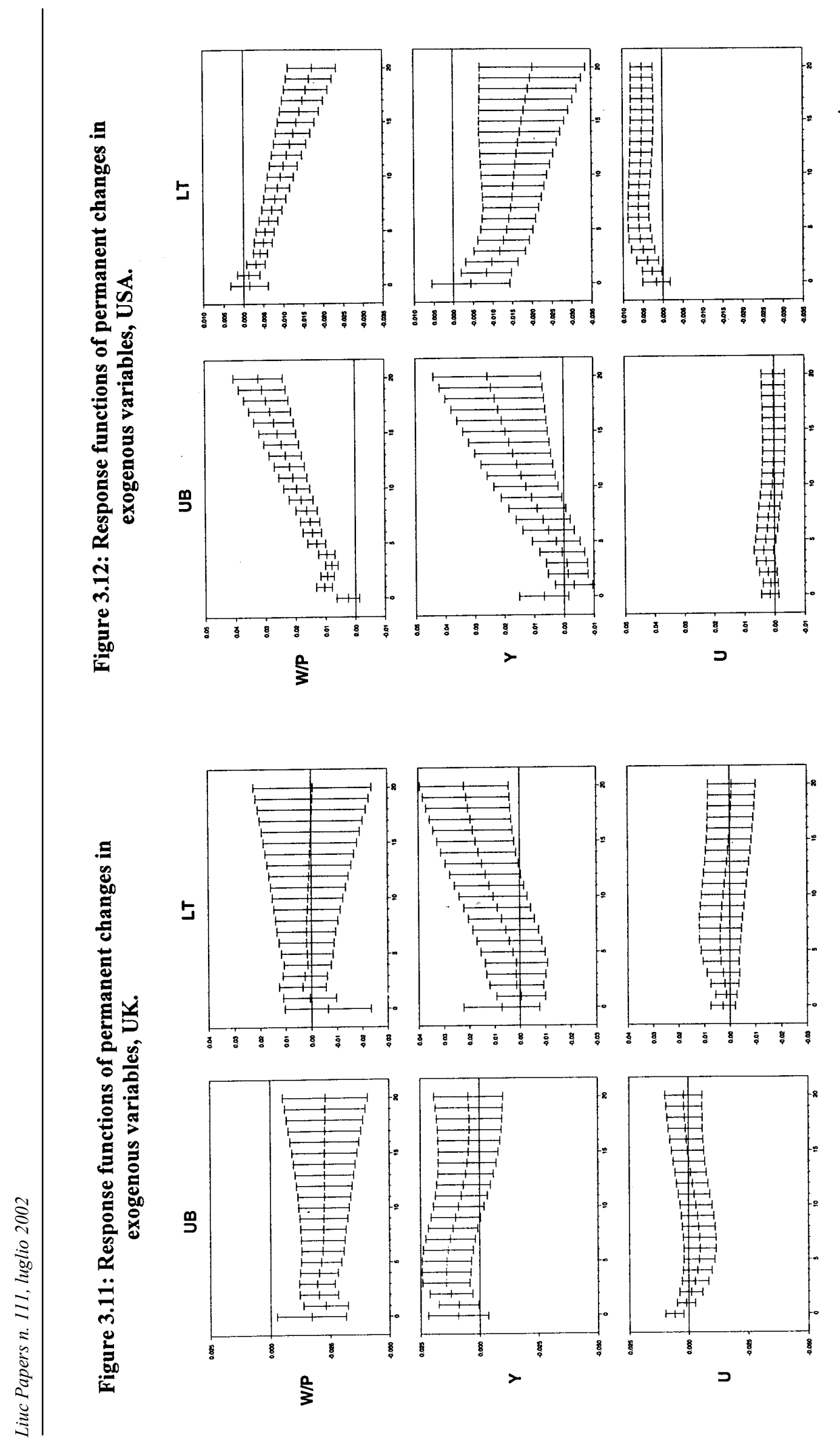

